\title{
Progressive failure and friction motion characteristics of contact surface of composite rock mass
}

\author{
Zhao Jinhai $^{1,2}$, Zhang Xinguo ${ }^{1,2}$, Pan Haiyang ${ }^{1,2}$, and Chen Juntao ${ }^{1,2 *}$ \\ 1 State Key Laboratory Breeding Base for Mining Disaster Prevention and Control, \\ ShandongUniversity of Science and Technology, Qingdao 266590, China; \\ 2 College of Energy and Mining Engineering, Shandong University of Science and Technology, \\ Qingdao 266590, China;
}

\begin{abstract}
The structural planes existing in natural rock mass can control the failure process of rock mass. Based on the progressive response-stability failure process of a single fracture interface, the relationship between progressive response and material failure of composite rock mass is discussed. The method of friction contact plane analysis in PANDAS numerical analysis software is applied to explore the correlation between the movement and mechanical properties of composite rock mass under external forces. The motion index is mainly represented by the sliding speed and distance of the contact surface, while the mechanical properties are mainly explained by the normal contact force, the friction of the contact surface, the friction coefficient and the material damage. The relationship between these six variables illustrates the progressive response relationship during the movement of the composite rock mass. Based on the static and progressive characteristics of the progressive response process of deep rock mass engineering, the failure evolution law and energy dissipation law of composite rock mass loading process and the synergistic failure characteristics of composite rock mass are discussed. The scientific problems that need to be studied in the structure, deformation and failure of deep rock mass are put forward. The reference is provided for the failure law of fractured rock mass in water inrush process under the influence of mining, as well as fault plate material and the study of mechanical state of rock mass in fault fracture zone.
\end{abstract}

\section{Introduction}

There are many kinds of contact interfaces in deep underground rock mass and underground engineering, such as different lithostratigraphic structural interfaces, geological fissures of the same lithology, and contact structural planes formed by other natural factors such as fracture zones and joints. When these structural contact planes are subjected to additional loads and pressure relief due to underground mining disturbances,

* Corresponding author: Zhao Jinhai, E-mail: jinhai.zhao@sdust.edu.cn 
the energy accumulation, dissipation and transfer process at the interface occurs under the influence of these contact planes. The stability of the system and the friction effect of the interface are closely related to the progressive performance of the interface. The results show that the stress wave reflects immediately when it reaches the energy transfer process on the interface, but accumulates energy instead of refracting immediately. When the accumulation reaches a certain degree, the energy transfer between the interfaces occurs suddenly, and the specific strain energy on both sides of the interface is much lower than that before the stress wave occurs. Therefore, the structural plane in the rock mass has the effect of accumulating impact strain energy and slowing the impact absorption. In addition, the displacement of the lower block has a great influence on the composite structure, and the displacement and internal force can be reduced. The research results of Wang (Wang.2007; Haeri et al.2014).

show that the compression wave velocity ratio of the upper and lower bodies is the main factor affecting the stress of the block system interface, i.e. the ability of the body to resist volume deformation. Therefore, studying the friction effect of the contact interface during the motion of the composite and the transient sliding and block failure characteristics of the interface are of great significance for studying the progressive instability process of the underground rock mass.

In the study of mechanical properties of rock mass with structural planes, Chen (Tan.1982, Tan and Kang.1991) realized that rock mass deformation consists of rock medium deformation in rock blocks and deformation of rock blocks in the vicinity of boundary planes (structural planes) and weakening areas (cracks) of rock masses, and carried out theoretical and experimental studies based on rheological viewpoints (Wang et al.2020, Guo et al.2001).Mu ( $\mathrm{Mu}$ et al.2013) analyzed the evolution law of physical parameters such as displacement, acceleration, stiffness and energy of each part of rock-coal-rock assemblage during loading. Liu (Liu et al.2014) used the improved Hopkinson bar to carry out experimental study on progressive failure characteristics of coal-rock assemblage under one-dimensional progressive and static loading. Zuo (Zuo et al.2018) put forward that coal-rock assemblage can improve the impact tendency of coal, and appraised the impact tendency of coal-rock assemblage in rock burst mine. Liu (Liu et al.2004) studied the mechanical properties and progressive failure characteristics of coal-rock assemblages with different height ratios through uniaxial tests. studied the overall strength and failure mechanism of monolithic coal, rock and coal-rock assemblages with 0 degree dip under uniaxial and triaxial loads were studied (Mohammadi and Tavakoli.2015; Panaghi et al.2015).

Potential failure modes of multi-fractured rock mass in nature are often manifested by the instability of rock blocks (blocks) formed by cutting by several structural planes and the free plane of rock mass (Hoek and Bray.2005, Yossef.2003; KUMSAR et al.2000; Komurlu et al.2016); The failure mode is wedge sliding. In order to explore the mechanism of instability and failure of multi-fractured rock masses, scholars have conducted a lot of research: (Wang et al.2007) studied the characteristics of failure movement of multi-fractured rock mass in near-fault mining, and analyzed the risk of progressive disasters. (Yuan et al.2012, Chen et al.2011, Ning.2009) studied the failure mechanism of rock mass near the free plane, and put forward the shear-tension failure mode of rock mass near the free plane, and the control method to control the stability of exposed rock mass. (Liu et al.2008) analyzed the failure characteristics of surrounding rock in near-fault mining, the mechanism and influencing factors of slip-line based on field measurement, carried out mechanics analysis of the failure of multi-fissured rock mass by using slip-line theory and obtained the dangerous range of failure. Zhao (Zhao et al.2020a; Zhao et al.2020b;) established the method system of block identification, block mobility and motion determination, block stability evaluation and block reinforcement analysis based on the 
finite element method, which provides a new way to study the stability evolution of block under various complex conditions.

Feng (Feng et al.2017)analyzed the progressive failure of a layer - crack structure based on the slabbing thin plate mechanical model. Zhao (Zhao et al.2018) reprocessed the failure process of the layer - crack structure through RFPA. Failure processes of the layer - crack structure influenced was simulated by progressive disturbance using LS - DYNA (Pen and Lu.2008). Zhao and Pan (Zhao et al.2019) researched the formation and failure mechanism of layer - crack structures according to model test results. Arora and Mishra (Arora and Mishra.2015) reproduced the failure process of layer - crack structures through biaxial or triaxial laboratory tests.

In order to study the corresponding relationship between fault and time, the development process of the damage factor, and the difficulty of monitoring the physical speed in the composite rock mass, such as monitoring speed and friction, computer simulation technology has become the most effective monitoring method. method. Starting from the progressive response-stability failure process of a single crack contact interface, the instantaneous changes of friction, sliding distance, sliding speed, friction coefficient and normal contact force during the loading process of composite rock are discussed. Quality, then get the main control and correlation between progressive response and material damage.

\section{Analysis of Mechanics Mechanism by Contact Model}

\subsection{Interlayer Slip Mechanism of Composite Rock Mass}

When analyzing the mechanical action of the interface between composite rock masses, considering the macroscopic properties of the interface, the interface of the composite rock mass is idealized as a horizontal interface (contact pair) in the continuous mechanical range, as shown in Figure 1(a). However, in order to preserve the effective information between the contact interfaces, it is necessary to consider the effects of normal stress and plane characteristics of composite rock mass interface on the shear strength and friction properties of the interface. Based on statistical analysis, Barton (Barton.1973) proposed the most widely used empirical equation of shear strength at structural level (JRS-JCS model) in 1973.

$$
\tau=\sigma_{n} \tan \left[J R C l g\left(\frac{J C S}{\sigma_{n}}\right)+\varphi_{b}\right]
$$

Where $\tau$ is shear stress, MPa; $\sigma$ is normal effective stress (axial loading force), MPa; JRC is roughness coefficient of structural plane; JCS is compressive strength of structural plane, $\mathrm{MPa}$; and $\varphi \mathrm{b}$ is basic internal friction angle of rock, $\left(^{\circ}\right)$.

Due to the contact surface in the composite rock mass, there is a tendency of relative sliding between the upper and lower contact faces of the two different rock blocks, and shear stress $\tau$ and friction stress $\mathrm{f}$ are generated at the contact faces. The positive and tangential deformation of the composite rock mass will occur under the axial load stress $\sigma_{\mathrm{n}}$, and the mechanism is shown in Figure 1(a). The normal displacement $u_{n}$ produced by the action of the stress $\sigma_{\mathrm{n}}$ on the contact surface of the overburden is expressed as an exponential (Yu et al.2018).

$$
u_{n}=u_{\max }\left[1-\exp \left(-\frac{\sigma_{n}}{k_{n 0} u_{\max }}\right)\right]
$$

Where $u_{\max }$ is the normal deformation of coal-rock interface, $\mathrm{m} ; \mathrm{k}_{\mathrm{n} 0}$ is the initial normal stiffness of coal-rock interface, $\mathrm{N} / \mathrm{m}$.

The relationship between the maximum structural plane closure $\mathrm{u}_{\max }$ was obtained (Bagheripour et al.2011), the initial normal stiffness $\mathrm{k}_{\mathrm{n} 0}$ of coal-rock interface and the 
roughness JRC of coal-rock interface, the compressive strength JCS of coal-rock interface and the initial opening $a_{j}$ by analyzing the closure test results of structural planes with different JRCs in 1983.

$$
\begin{gathered}
u_{\max }=H+I(J R C)+J\left(\frac{J C S}{a_{j}}\right)^{k} \\
\mathrm{k}_{n 0}=-7.15+1.75(J R C)+0.02\left(\frac{J C S}{a_{j}}\right)
\end{gathered}
$$

Where ,H, I, J and $\mathrm{K}$ are all regression coefficients, and $a_{j}$ can be estimated by formula (5).

$$
\mathrm{a}_{j}=\frac{\mathrm{JRC}}{5}\left(0.2 \frac{\sigma_{C}}{J C S}-0.1\right)
$$

Where, $\sigma_{\mathrm{c}}$ is uniaxial compressive strength , $\mathrm{MPa}$ 。

When the value of $\tau$ is greater than the value of $f$ (satisfies the value in equation (6)), the sliding motion between rock blocks occurs and horizontal sliding deformation occurs in the composite rock mass (Fig. 1 (a)).

$$
\tau>=f=\sigma_{n} \tan \left[J \operatorname{RClg}\left(\frac{J C S}{\sigma_{n}}+\varphi_{b}\right)\right]
$$

Where, $\sigma_{n}$ is the axial stress, $\mathrm{MPa}, \varphi_{\mathrm{b}}$ are the basic internal friction angle of rock, $\left({ }^{\circ}\right)$

$\circ$

Under the action of compressive shear load, the shear-slip deformation between the contact faces of the composite rock mass includes plastic deformation and brittle deformation, and slip along the contact surface will occur.

After the shear failure of the structural plane, a sliding motion will be formed between the rock masses. At this time, the main control weak plane (contact plane) controls the friction and sliding instability of the sliding body. The coefficient of friction and cohesion of the structural plane play an important role in the motion of the composite. The progressive load shock formed by the relative sliding speed between the contact blocks is of great significance to the failure mode and process of the composite rock mass.

\subsection{Nonlinear numerical simulation method of friction contact}

In composite rock mass, the mechanical properties of rock blocks are closely related to the characteristics of the contact plane. The most representative aspect is the treatment of contact boundary conditions. The most influential work is to consider the contact problem as a constrained minimum value problem, and to solve the minimum value problem by combining the mathematical programming method with the finite element method. Usually, the penalty finite element method is used to solve this problem. The non-embedded condition of the contact area is introduced as a weighting term into the total potential energy of the contact system, and the constrained variational problem is transformed into a penalty optimization problem. Compared with the finite element method of mathematical programming, the advantage of the punitive finite element method is that it only needs to solve the node displacement vector without considering the contact force, thus reducing the calculation amount (Yao et al.2018). The essence of contact problem in rock mass system is the synthesis of boundary nonlinearity, material nonlinearity and geometric nonlinearity in the process of stress concentration. In contact research problems, the two planes of contact are usually named subordinate plane and dominant plane. As shown in Figure 1 (b), $\mathrm{B}^{1}$ is subordinate plane and $\mathrm{B}^{2}$ is dominant plane. The dominant plane should include the subordinate plane completely. The solution is carried out in a point-to-point manner, that is, a subordinate point can only correspond to one point on the dominant plane, but a point on the dominant plane can correspond to one or more nodes on the subordinate plane at the same time. This is the theoretical basis of Node-to-Point contact unit (Valkó and Economides.1994, W. brace.1966, Xie.1966, Liu.2014). 


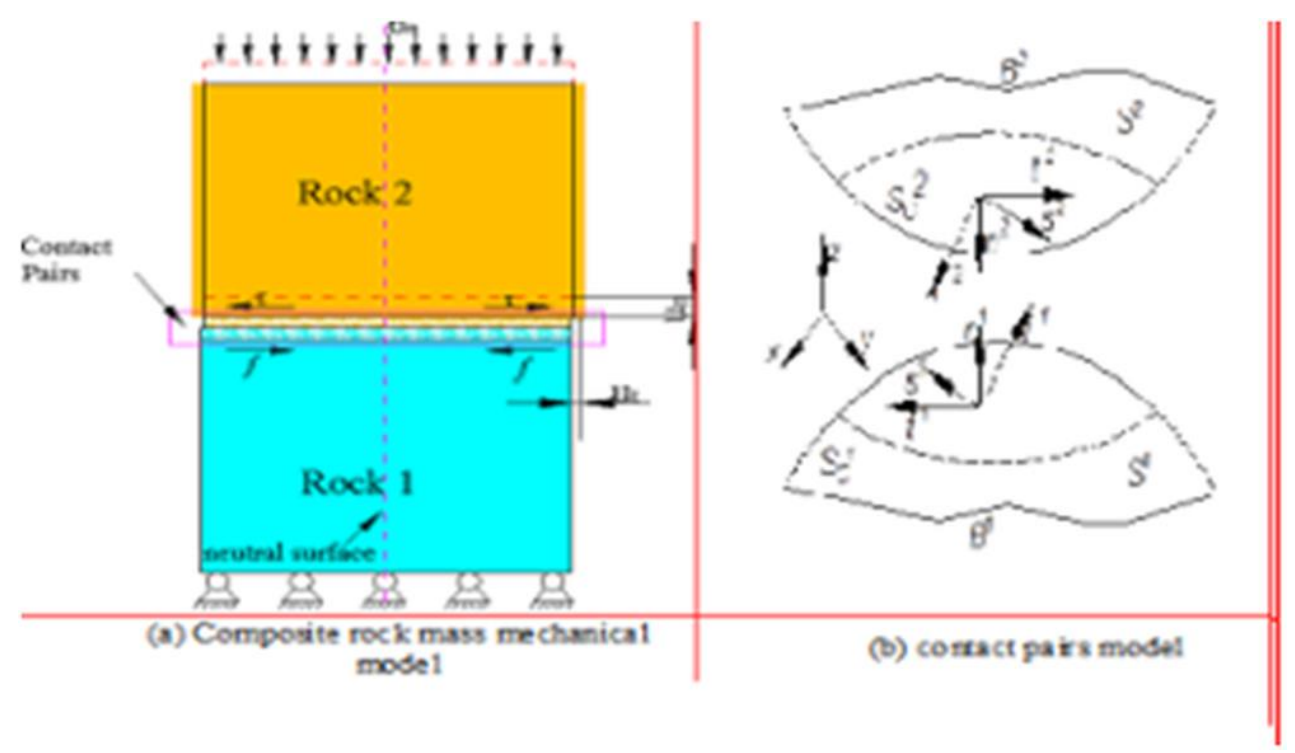

Fig. 1. Composite rock mass mechanical model and contact pairs model

The mechanical analysis model of each contact point in Fig. 1 (a) is shown in Fig. 1 (b), in which $\mathrm{B}^{1}$ and $\mathrm{B}^{2}$ are two contacts, $\mathrm{S}^{1}$ and $\mathrm{S}^{2}$ are contact planes, and $\mathrm{S}_{\mathrm{c}}$ is the intersection of two contact planes $\mathrm{S}^{1}$ and $\mathrm{S}^{2}$. $\mathrm{N}$ is the normal direction of the contact plane, $\mathrm{s}$ is the tangent direction of the contact plane, $\mathrm{f}$ is the traction force on the contact plane, $\mathrm{t}=\mathrm{n} \times \mathrm{s}, \mathrm{s}$ and $\mathrm{t}$ together constitute the tangent plane of the contact plane. In this paper, the penalty function method is used to establish the relationship between normal contact forces between contact pairs (Xing et al.2007, Song et al.2018). When $\mathrm{g}_{\mathrm{n}}<0$, contact occurs.

$$
f_{n}=f \cdot \pi=E_{n} g_{n}
$$

Where, $\mathrm{f}_{\mathrm{n}}$ is the normal contact force, $\mathrm{E}_{\mathrm{n}}$ is the penalty factor in the normal direction, $g_{n}$ is the normal penetration distance, $\pi$ the external normal direction of the contact surface. $g_{n}=\pi \times\left(x_{s}-x_{c}\right), x_{s}$ and $x_{c}$ are the coordinate positions of subordinate points $\mathrm{s}$ and their corresponding contact points, respectively.

\subsection{Nonlinear Finite Element Method for Friction Contact}

The updated Lagrangian formula is used to solve nonlinear problems. In the form of virtual velocity, the equilibrium equation can be expressed as (Xing et al.2007, Song et al.2018)

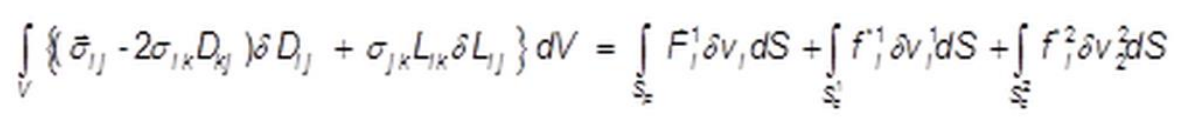

Where, $V_{i}, S$ - $\mathrm{t}$ the volume and surface area of deformed body $\mathrm{B}$ at time $\mathrm{t}, S_{F}$ - the action boundary of load $\dot{F}_{i}$ on surface $\mathrm{S}, \delta v_{i}$ - the virtual velocity field, On the Velocity Boundary when $\delta v_{i}=0, \bar{\sigma}_{i j}$-stress ratio, L-Velocity gradient tensor ( $L_{i k}=\partial V_{i} / \partial x_{k}, \delta L_{i j}$ is virtual velocity gradient tensor) $\dot{f}_{i}$-Change Rate of Contact 
Force on Sc Contact Surface, $\mathrm{V}_{2}$-Relative sliding velocity between contact pairs , D、W-the symmetric and antisymmetric parts of tensor (Xing et al. 2007, Song et al. 2018, Song et al. 2018).

\subsection{Introduction of numerical simulation software PANDAS}

The above numerical simulation methods of fault friction and contact are integrated into the numerical simulation software PANDAS (Parallel Adaptive Nonlinear Deformation Analysis System). PANDAS is an innovative software platform (Xing et al.2007, Song et al.2018, Song et al.2018) based on the finite element method (FEM) and lattice Boltzmann method (LBM). It has been successfully used to simulate highly nonlinear coupling of multiphysics in heterogeneous porous materials/stratigraphic layers with subsurface fractures, such as "stress deformation/fault-fluid flow-heat conduction-chemical reactions" and solves major scientific problems (Selcuk.2019). And engineering challenges in geoscience and earth resource engineering related to micropore size, laboratory scale, oil and gas field size, and even global scale.At present, the software uses self-adaptive staticprogressive algorithm to simulate the progressive and evolutionary process of fault system, taking different progressive phenomena into account, such as adhesion and slip state on contact surface and state change between them. It has a wide influence on the study of mechanical properties of contact rock mass.

\section{Progressive response analysis of single fracture}

In order to accurately monitor and control the coordination between the slip surface and the material properties, in particular to solve the corresponding relationship between fault and time, the development process of the fault unit and the difficult problem of monitoring the slip speed, friction and other physical quantities on the surface, the computer Simulation technology has become the most effective monitoring method. From the progressive response-stability failure process of a single crack, the frictional force, sliding distance, sliding speed, friction coefficient and instantaneous contact force of the contact surface under different inclination angles and different inclination angles are obtained. The material parameters between the upper and lower rock masses and the motion process of the composite rock mass are discussed. The main control relationship between progressive response and material damage is obtained.

\subsection{Experimental study on fracture process of contact rock mass}

In order to restore the destructive properties of contact rock mass under natural conditions and better integrate with the actual situation, this paper adopts the method of composite rock mass to carry out laboratory experiments. According to the rock mechanics parameters obtained from the single loading experiment of each rock mass, the composite rock mass is obtained by the overall loading of the composite rock mass. The overall strength and mechanical parameters of the composite rock mass. Experimental rock samples are shown in Figure 2. The length, width and height of the sample are both $50 \mathrm{~mm}$.

The single and multi-body loading experiments are carried out by the Shimadzu AG-X250 testing machine. The maximum experimental load is $250 \mathrm{kN}$. The machine has the characteristics of good stability and high accuracy. The displacement loading control is adopted in the experiment. The loading process continues until the sample is destroyed, and 
after reaching the peak, the axial force of the rock will quickly disappear. At the same time, the shape and loading speed of the rock mass after the experiment were retained. The rate is set to $0.005 \mathrm{~mm} / \mathrm{s}$. During the experiment, the computer synchronously collected experimental data with a sampling interval of $0.5 \mathrm{~s}$. The stress-strain curves for uniaxial and composite rock masses in uniaxial compression and failure modes are shown in Figure 3-5. Three groups of identical specimens, four in each group, were allocated in the experiment. For the first group, the numbers are 1-1, 1-2, 1-3, 1-4, respectively. In group 1-1 and 1-2, uniaxial compression tests are carried out to determine the strength of the specimens, while in 1-3, 1-4 rock mass is carried out to test the properties of the composite rock mass, and the contact surface of the composite rock mass is polished to ensure the surface accuracy, and the static friction coefficient is 0.6.

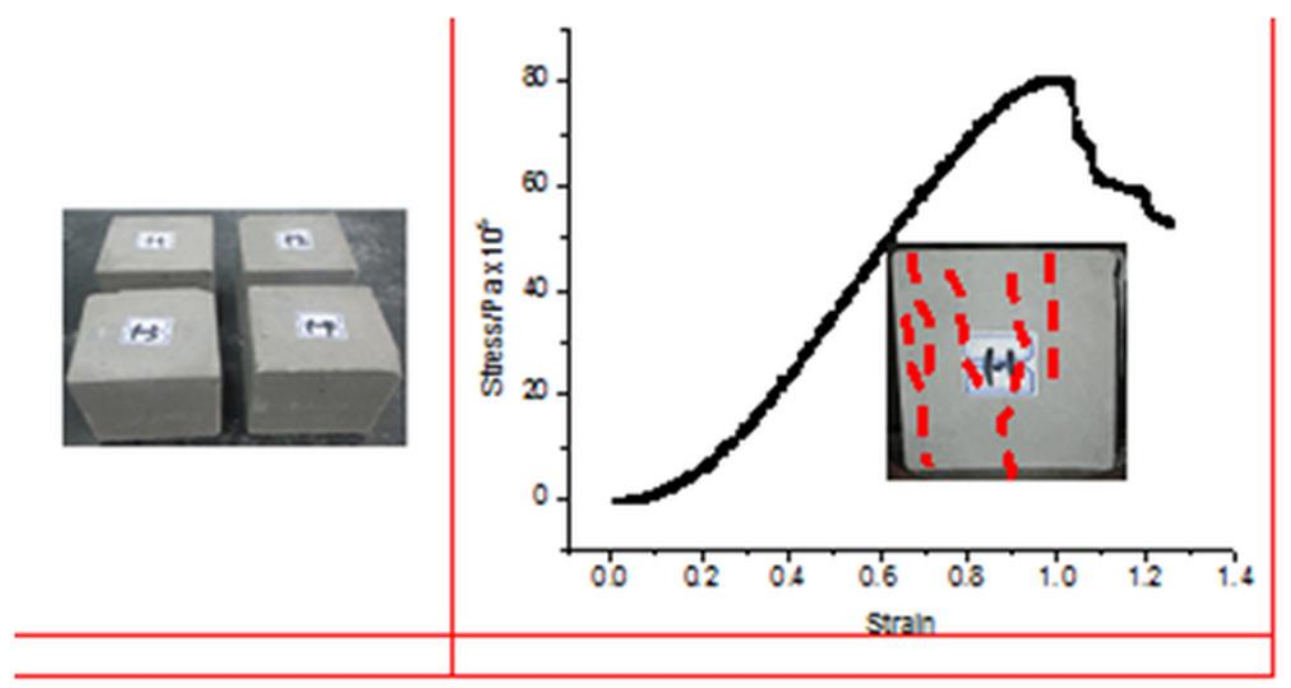

Fig. 2. Prepared rock-like blocks

Fig. 3. Uniaxial compression stress-strain curve of test piece 1-1

In the experiment, the splitting failure is the main factor in the uniaxial compression test of monomer. Under the same experimental conditions, the failure modes are basically the same, and the difference in rock mass strength is small. The maximum strength values are 8 $\mathrm{MPa}$ and $8.5 \mathrm{MPa}$, respectively. There are two stress mutations in the experimental process of composite rock mass, which correspond to two crack propagation processes. As shown in Figure 5, double shear failure is the primary mode of failure of the composite. There is a nuclear zone in the interface of the composite. Affected by the middle contact surface, although the material properties of the upper and lower rock masses are the same, the upper rock mass is mainly destroyed at the edge and corner of the material, and the internal failure of the rock mass mainly occurs in the lower rock mass. 


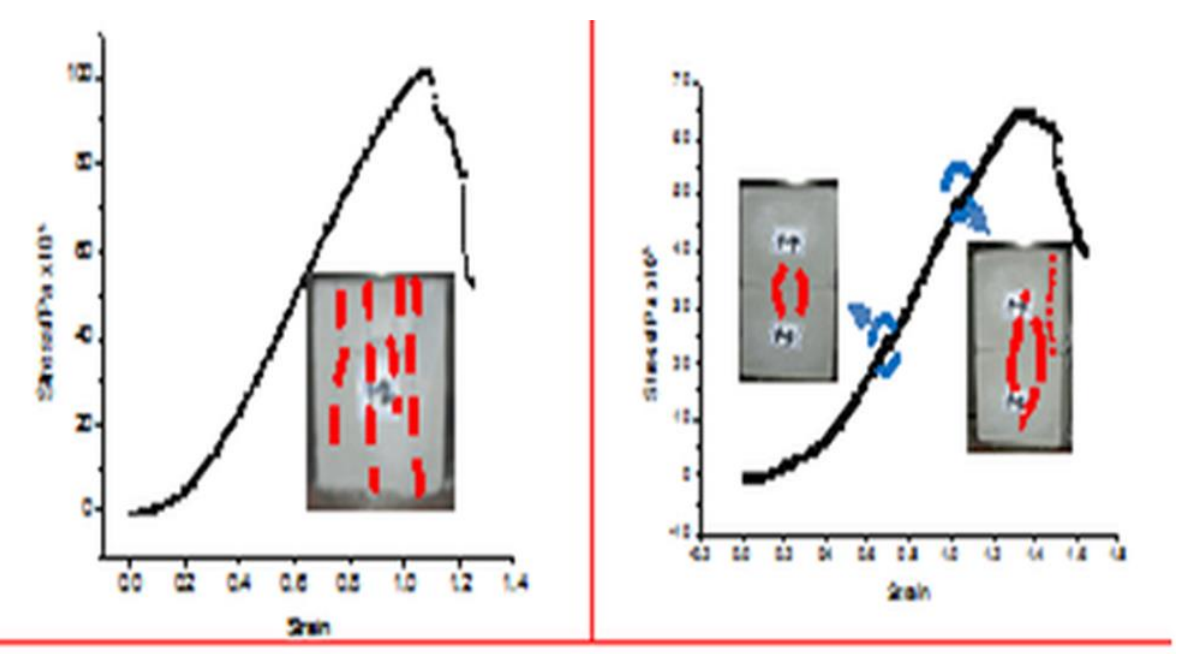

Fig. 4. Uniaxial compression stress-strain

Fig. 5. Compressive stress-strain curve of curve of test piece 1-2 combined rock mass test pieces 1-3,1-4

\subsection{The numerical model and parameters of transverse fracture}

The contact model is used to analyze the numerical model of composite rock mass. By comparing the numerical simulation results with the experimental results, the rationality of the contact pair model in simulating the deformation, slip and fracture of composite rock mass is verified. In the model, the displacement in the bottom three directions is fixed and the applied load on the top is $20 \mathrm{MPa}$. The contact positions of the two blocks are connected by contact pairs, and the other boundaries are free boundaries. The grid and boundary condition settings for the numerical model are shown in Figure 6 . In the numerical simulation, the composite rock mass specimen is $50 \times 50 \times 100 \mathrm{~mm}$, the same size that of the experimental specimen, the size of which is, and it is divided into two blocks, each size is $50 \times 50 \times 50 \times 50 \mathrm{~mm}$, and each block is divided into $16 \times 16 \times 10$ computational mesh elements. The initial static friction coefficient is 0.6 on the contact surface of the two blocks, referring to the experimental results of literature (Xing et al.2007, Song et al.2018, Song et al.2018, Xing et al.2000, Feng et al.2019, Feng et al.2020) and practical experience parameters is set: $\mathrm{a}=0.01, \mathrm{~B}=0.025$. The Mohr-Coulomb criterion is used as material yield criterion. The upper and lower blocks adopt the same material mechanical parameters, as shown in Table 1. Constant boundary pressure loading is adopted, and R_min loading mode is used to simulate the transient force loading. The self-adaptive nonlinear solver automatically selects the calculation step size and controls the stress of each step until the boundary force loading is completed. 


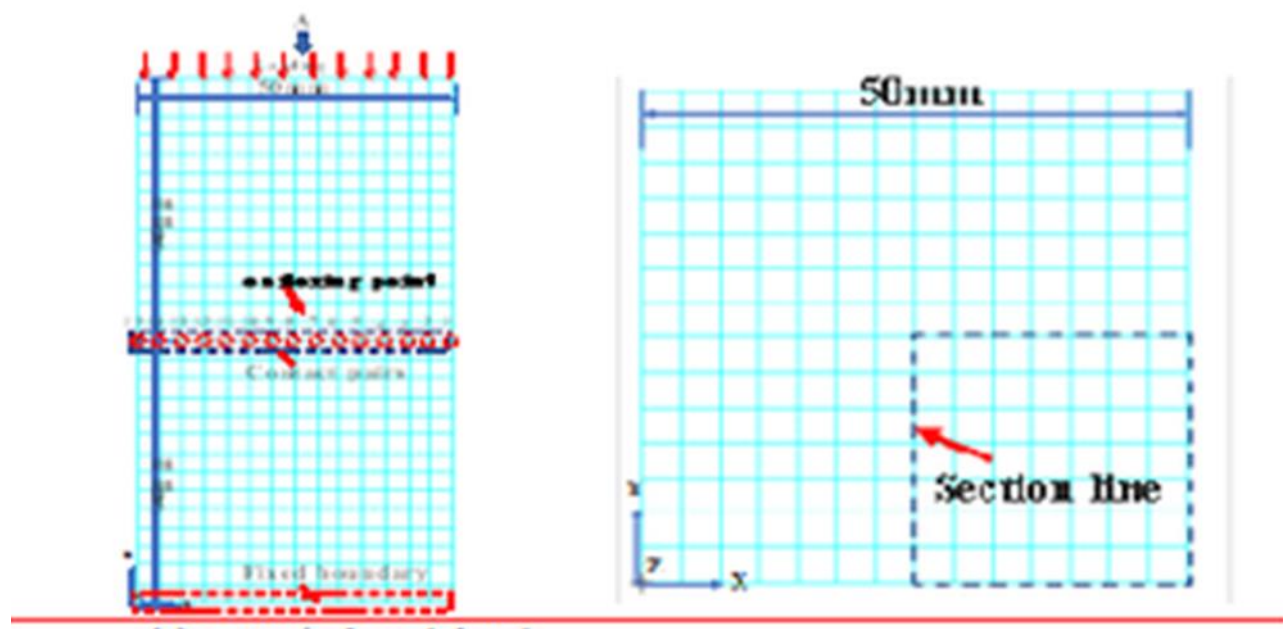

(a) numerical model and

boundary conditions

(b) A-Directional View

Fig. 6. Combined rock mass numerical model grid and boundary conditions

Table 1. Mechanical parameters of calculation model

\begin{tabular}{|c|c|c|c|}
\hline Elasticity modulus/MPa & Poisson's ratio & Yield stress /MPa & Internal friction angle \\
\hline 10450 & 0.35 & 6.74 & 20.8 \\
\hline
\end{tabular}

\subsection{Transient Loading Law of Transverse Fracture Model}

In order to analyze the stress evolution law of composite rock mass during time loading, this paper chooses the results of stress variation at different loading stages (representing different loading stages), in which step represents the calculation steps, related to loading time. Figure 8 shows the stress change process of the numerical calculation model of composite rock mass under axial load. It can be seen from the figure that the stress concentration phenomenon first appears at the corner of the bottom boundary, and a stress concentration region is formed near the fixed boundary. Then, the corner position is broken, and the stress concentration region at the bottom is further enlarged to form a small-range saddle stress concentration region. The stress concentration region is also formed near the boundary of the composite contact surface, eventually forming a fracture region. As the axial force is further loaded, the bottom stress concentration region is connected to the upper stress concentration region (step 16 in Fig. 8). At the same time, the failure direction of the failure area at the contact surface is differentiated, and extends to upper and lower combination monomers respectively. In the lower rock mass, an independent failure area is formed first, which accelerated the failure process of the material body in the lower rock mass(step 26 in figure 8). With further stress loading, the new failure location is located in the rock mass between the failure areas of the lower rock mass. The failure areas are connected through the "minimum path" (step 27 and step 28 in figure 8). 
Fig. 7. Stress-strain curve of monitoring point 1
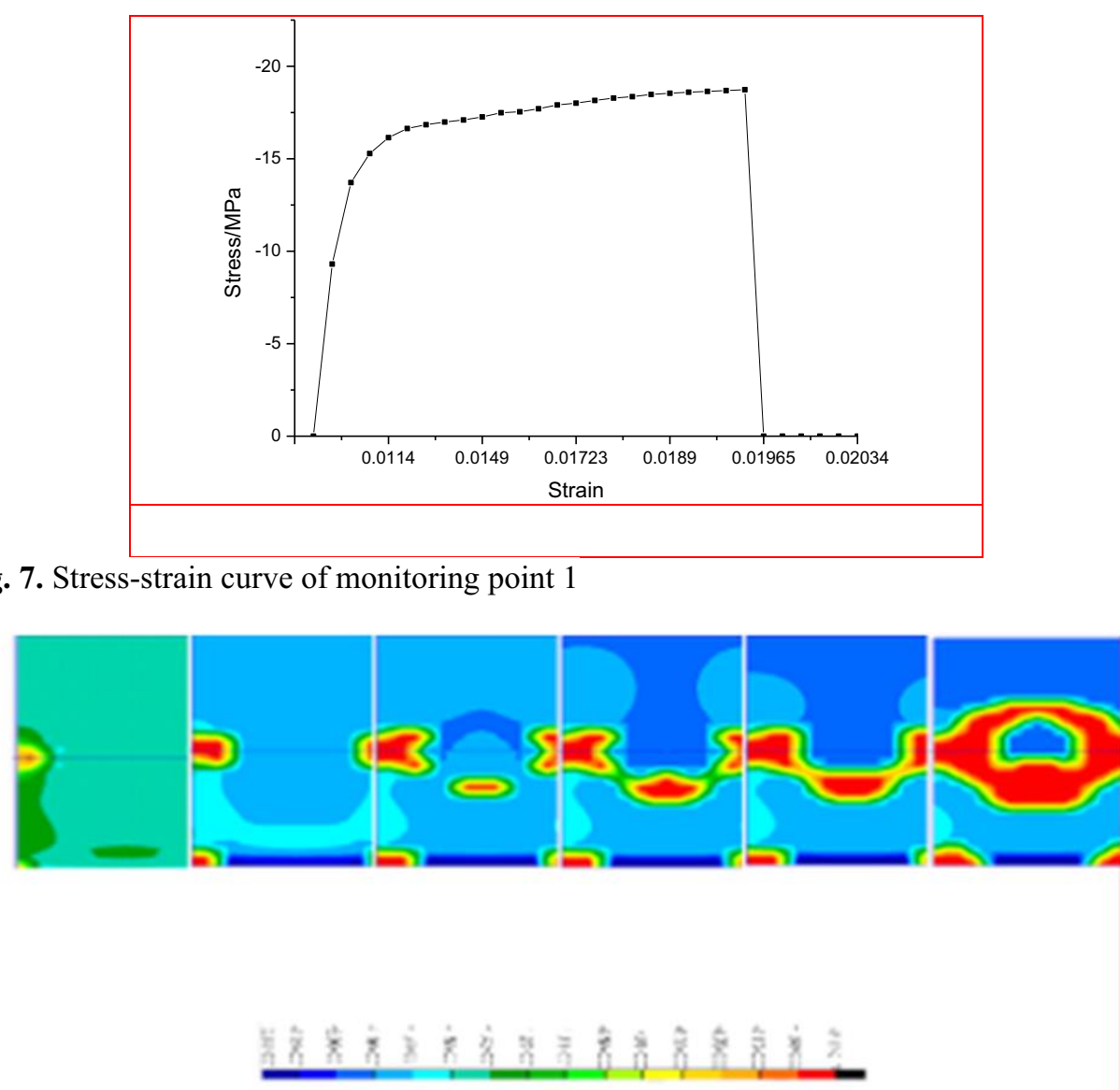

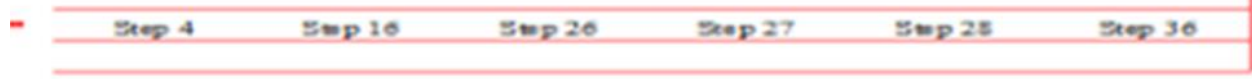

Fig. 8. Stress Evolution Process of Composite Rock Mass Numerical Model

Under the influence of material damage and energy release in the lower rock mass, the material failure development in the upper rock mass is relatively slow, and the material failure direction in the upper rock mass continues to develop along the original failure direction. The stress concentration zone is formed in the middle of the two failure zones of the upper rock mass, while the stress concentration near the boundary is low. In the middle of the contact surface, there is a stress-concentrated core area, but the material has not been destroyed. With the further increase of the axial force, the upper rock mass continues to develop along the direction of the formation of the failure and connects within the upper rock mass, which ultimately leads to the overall instability of the rock mass, while the core area formed in the rock mass has not been destroyed (s in Fig. 8). Step36).

The above simulation results are the same as those observed in experiments, and the change process inside rock assemblage can better reflect the essence of transient process change. From Figure 9, it can be seen that the change of the above boundary is an indirect reflection of the stress evolution process on the boundary. The simulation model is cut off by a quarter 
to study the stress change process inside the rock block assemblage. The failure of the rock mass begins at the bottom boundary and the contact boundary. However, by the connection of the upper and lower stress concentration regions, isolated stress concentration points are formed in the lower rock mass, which leads to the destruction of the rock mass material. During the formation of the damaged area, the damaged area on the boundary of the rock mass and the damaged area in the rock mass are independently developed. The interaction is small, the damage process develops slowly, and the stress value rises rapidly, and the strain value is small.

With the loading of axial force, new independent failure zones are no longer generated, but interconnection of destroyed areas are mainly taking place. At this time, the interaction between the failure zones is greater than the independent stress change trend of the failure zone itself. It can also be seen from the stress change process of monitoring points that the stress value increases slowly down from the original sudden in previous stage, and the change of stress value is small, mainly due to the deformation and failure of materials. The failure development of the material itself in the lower rock mass also takes precedence over the development of the contact material.

The failure of lower rock mass has undergone independent development of internal and external failure zones, and ultimately the failure zones interact with each other and connect with each other on the principle of the nearest path, forming the whole process of instability of lower rock mass. There are still unspoiled core zones between the contact surfaces. From the summary of failure priority, it can be seen that rock mass corner failure $>$ internal independent failure of lower rock mass $>$ regional failure between failure zones $>$ inward failure of contact to edge $>$ upper rock mass failure $>$ contact to nuclear area failure. The above variation law corresponds to the principle of synergistic failure in rock mass failure. Synergy is used to explain the macroscopic behavior that causes the spontaneous formation of space-time structure and its special functions in complex open systems. The stress change in the specimen first proceeds independently with a certain mechanical law, and each block develops independently in accordance with the field and forms its own nuclear area. Then the nuclear area expands continuously, and finally through the minimum path, connects with each other to reach the unstable state (Vyazmensky and Elmo.2010, Prudencio and M van.2007; Gomberg et al.2003).
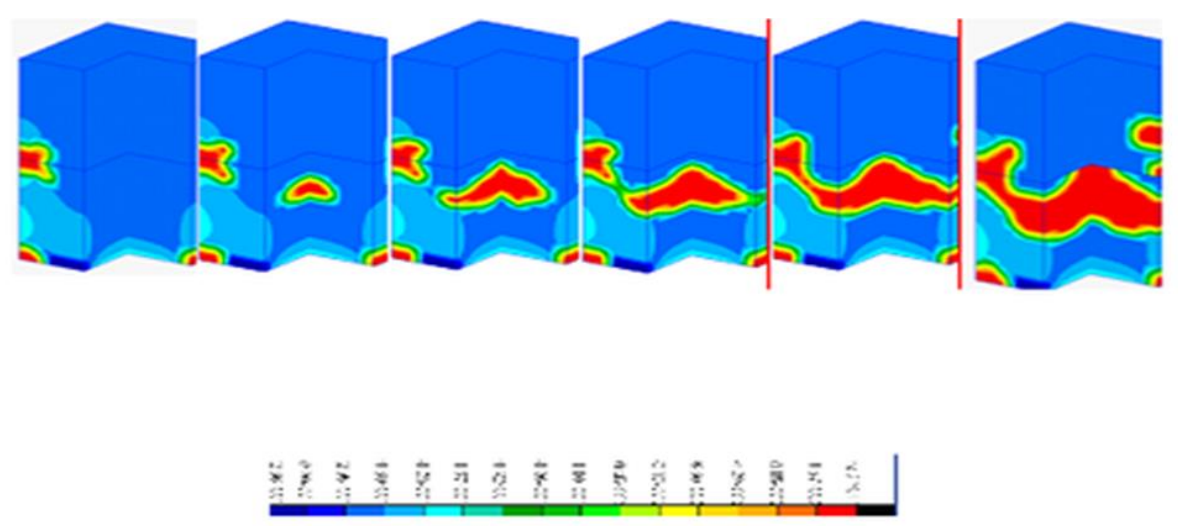

\begin{tabular}{llll|l|l}
$\operatorname{Step} 20$ & $\operatorname{Step} 22$ & $\operatorname{Step} 26$ & $\operatorname{Step} 27$ & $\operatorname{Step} 30$ & Step 34
\end{tabular}

Fig. 9. stress evolution processes of numerical model 


\section{Discussion}

In this paper, a non-contact model is established as shown in Fig. 10, and the differences of material damage and stress changes between the two models are compared. Under the condition of uniaxial loading near fixed bottom boundary, the failure of composite rock mass mainly occurs in the interior of materials with lower strength. When two simulation methods are adopted, the edge and corner positions of weak rock mass materials are first destroyed, and the main failure mode is compression-shear failure. The difference is that when the contact pair is not analyzed, material failures are formed from the inside and progress from the initial fault area to the surrounding area. The initial failure zone is formed in the lower center of the interior of the material, but the development direction is mainly upward and four sides. Due to the limitation of the fixed boundary at the bottom, the failure zone develops slowly to the bottom, and eventually the failure of the rock mass extends to the top surface and causes the material to completely destabilize.

\subsection{Analysis of non-contact simulation results}

Compared with non-contact model and contact pair model, it can be seen that the existence of contact surface has a strong control effect on the failure of rock block assemblies. The formation and expansion of material failure zone mainly concentrates near the contact surface, and the end point of the contact surface as the initial failure location extends to the inside of the block, and inter links with the failure zone formed inside the rock block. By comparing the experimental phenomena, it can be seen that the simulated failure law of the contact pair is the same as the experimental results, but the simulated results of the non-contact pair are quite different from the experimental phenomena, so it is more reasonable to use the contact pair to simulate.

By comparing the failure sequence and failure law of composite rock blocks, it can be seen that under the influence of fixed boundary conditions at the bottom, although the material properties of upper and lower rock masses are identical, the failure of coal-rock combination first occurs in the lower rock mass and then extends to the upper rock mass. This process corresponds to the experimental phenomenon (Xing et al.2007, Song et al.2018, Song et al.2018,). The reason can be explained as follows: a large amount of energy is released in the process of unit rupture, and some of it is transferred to the adjacent unit, which will accelerate the destruction of surrounding units. At the same time, stress relief after failure of the joint will cause the surrounding joint to lose its confining pressure constraints and destroy surrounding components and create a chain effect. In a composite rock mass, the two contact blocks are joined by a frictional contact pair. During axial force loading, the nodes release energy by deformation and sliding motion around the joint, thereby reducing the force transmitted from the axial load to the lower rock mass. At the same time, the release of energy in the contact deformation can inhibit the destruction of the bulk material itself, which will increase the bulk strength in the form of a phase change. The effect of sliding deformation on the deformation resistance of the slider will be explained in detail in the inclined contact slider.

Comparing the simulation results of the internal and external changes of the specimens and the experimental results of the combination, it can be seen that the experimental results are highly consistent with the simulation results, which also verifies the rationality of the numerical analysis software. The main common points lie in the following three aspects: (1) The development of failure zone is independent from the corner position and the inside of the specimen, but the joint process between the failure zone formed independently in the lower rock mass and the boundary failure zone is the real cause of instability failure of the 
composite rock mass. (2) Although the stress concentration in the interior of the block is higher than that in the margin, the non-failure core area is formed because of the confinement of surrounding rock mass and the transfer and release of contact forces to both sides. (3) The failure time and scope of the upper block are less than those of the lower block, that is, the failure of the upper sliding disk is later than that of the non-sliding disk.
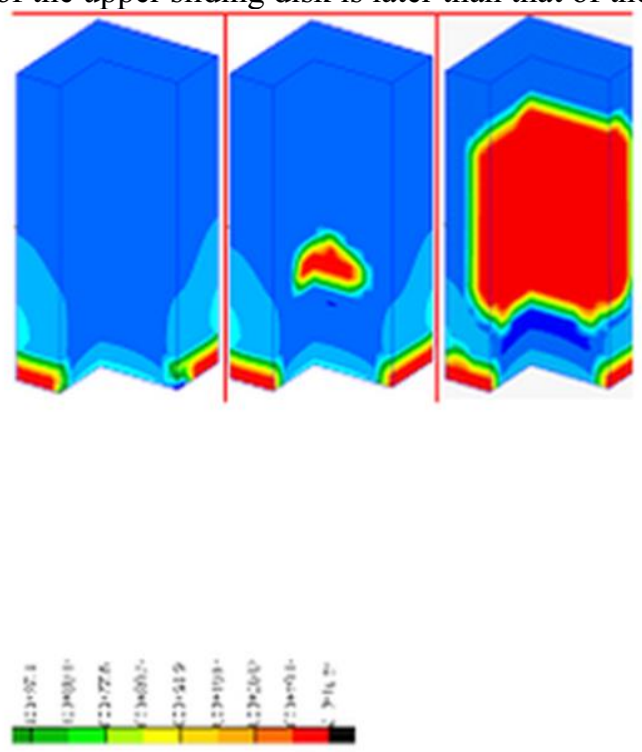

\begin{tabular}{l|l|l}
\hline Sep 14 & Step 20 & Scep 49 \\
\hline
\end{tabular}

Fig. 10. Variation of vertical stress without contact pair

\subsection{Study on Progressive Performance of Contact Surface}

The material parameters of rock mass assemblage monomer are identical, but the failure modes of rock mass assemblage monomer are obviously different due to the influence of the middle contact pair and the fixed boundary at the bottom. The upper block of the composite rock mass releases energy during horizontal sliding and deformation (Fig. 11 Fig. 15), which reduces the damage to the upper rock mass itself, so the damage of the upper rock mass develops relatively slowly. 


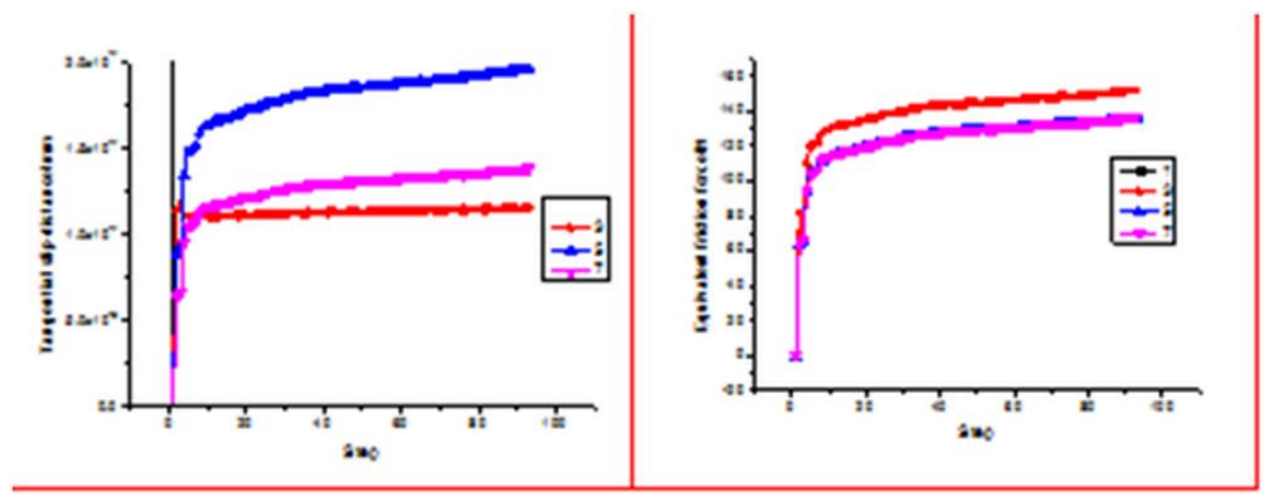

Fig. 11. Varibation of tangential displacement

Fig. 12. Varibation of equivalent friction

During the initial phase of loading, the friction and sliding distance can vary greatly. For nodes near the boundary (such as node 1), the displacement will increase gradually and the friction will increase relatively slowly. For the nodes near the center, as shown in the monitoring nodes 3, 5 and 7 in Fig. 6, the friction force increases step by step and the relative displacement increases slowly, which indicates that the corner position on the contact surface produces lateral deformation under the action of axial force, while the horizontal sliding friction occurs due to the inconsistency of movement between contact surfaces due to different constraint conditions of the upper and lower parts of the composite rock mass.

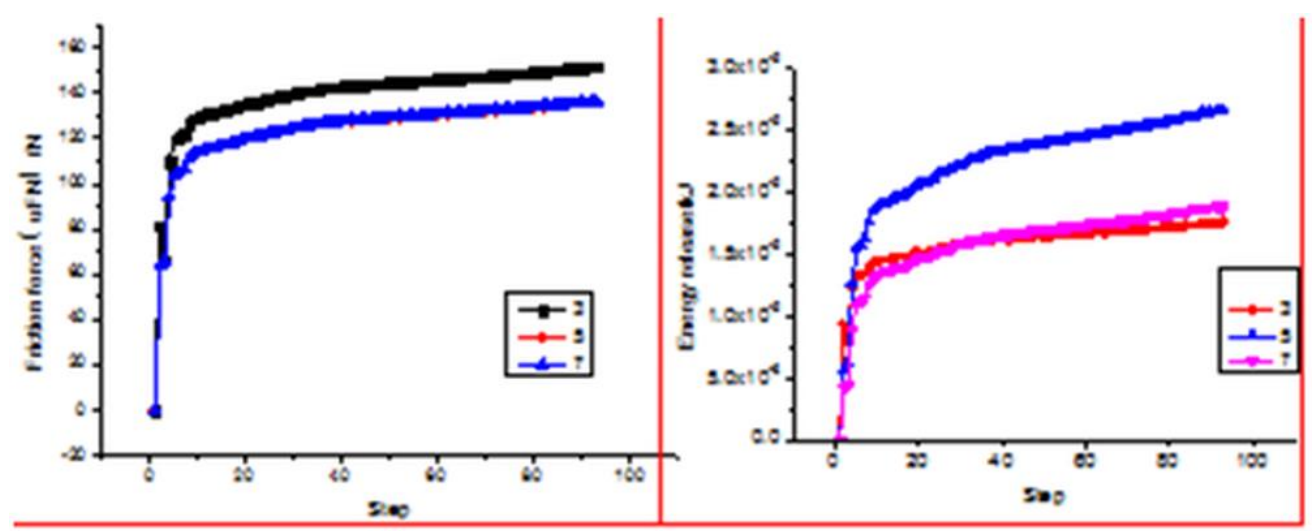

Fig. 13. Chang rule of friction force

Fig. 14. Energy loss variation curve of the monitoring point

\subsection{Analysis of Displacement Characteristics of Contact Surface}

The contact force of internal joints increases, and the contact surface is dominated by static friction. Therefore, the sliding displacement between the inner and outer joints is 
completely different, which results in a large tension area between the edge joint and the intermediate joint, while the other side of the edge joint is a free surface, which does not provide lateral force constraints. This is also one of the reasons why the damage scope of the contact surface is larger than that of the fixed restraint position at the bottom, and why the corner position is easy to be destroyed during the deformation of composite blocks. The displacement distribution law is shown in Fig. 15.
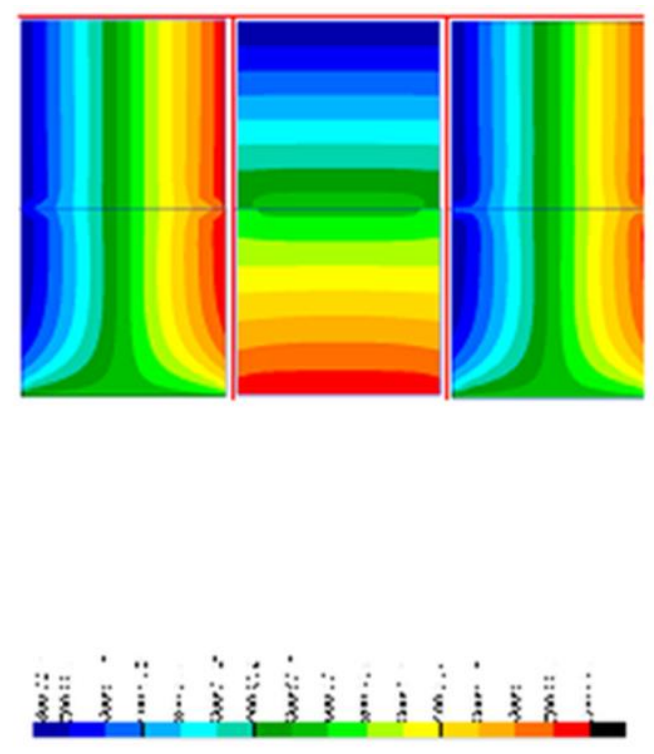

\begin{tabular}{l|l|l}
\hline $\mathrm{X}$-direction & y-direction & $\mathrm{z}$-direction \\
\hline
\end{tabular}

Fig. 15. Cloud picture of displacement in different directions

\section{Conclusion}

Based on the lateral contact composite rock mass, the mechanical load test of the composite rock mass was carried out. The overall mechanical law of the composite rock mass from local independent failure to instability near the contact surface is obtained. The rationality of numerical simulation of composite rock mass using contact pair is verified by comparison with numerical simulation method.

(1) There is a non-destructive nuclear zone between the contact faces of the composite rock mass. The material parameters of the upper and lower parts of the assembly are the same and the lower part is the fixed boundary. The failure time and range of the bottom fixed disk is better than the failure time and range of the upper sliding disk. This process corresponds to the experimental results.

(2) The numerical model is used to analyze the sliding and material failure characteristics of rock masses with different dip angles. The results show that the failure time and range of the sliding disc are smaller than that of the non-sliding disc, and the transient changes of 
friction, normal contact force, relative motion speed and sliding distance on the contact surface are obtained.

(3) It can be seen from the failure law of the numerical model of transverse fracture that the failure of the model occurs near the contact surface, and the existence of the contact surface has a controlling effect on the failure process. The formation of the destructive zone has changed from independent development to mutual penetration, which is in line with the cooperative destruction process.

\section{Acknowledgments}

Funding was provided by National Key R\&D Program of China(2018YFC0604705); SDUST Research Fund (grant 2018TDJH102); National Natural Science Foundation of China (Grant Nos. 51574159 51974172, 51974173, 51804179) and the SDUST Research Fund, Shandong Province Natural Science Foundation Project (ZR2017MEE055 ; 2018GSF116002;2019GSF111024); China postdoctoral science foundation (2015M572067,2016T90662.

\section{References}

1. S. Arora and B. Mishra, Int j rock mech min sci, 79, 109 (2015)

2. M. Bagheripour, R. Rahgozar, H Pashnesaz, Geomech., 3:1, 61 (2011)

3. N. Barton, Engineering Geology, 7:4, 287 (1973)

4. L Chen, X.R. Meng and Z.N. Gao, Coal science and technology, 39:5, 18 (2011)

5. F. Feng, X.B. Li, D.Y. Li and S.F. Wang, Chin J Geotech Eng, 39:7, 1302 (2017)

6. F. Feng, S.J. Chen, D.Y. Li, S.T. Hu, W.P. Huang, B. Li. Energy Science and Engineering, (2019)

7. F. Feng, X. Li, K. Du, International Journal of Geomechanics (2020)

8. D.M. Guo, J.P. Zuo, Y. Zhang and R.S. Yang, Rock and soil mechanics, 32:5, 1333 (2011)

9. J, Gomberg, P. Bodin, P.A. Reasenberg, Bulletin of the seismological society of america, 93:1, 118 (2003)

10. E. Hoek, J.W. Bray, Rock slope engineering, 23, 125 (2005)

11. H. HaeriShahriar, M. F. Marji, Int. J. Rock. Mech, 67, 20 (2014)

12. H, Kumsar, O. Aydan, R, Ulusay, Rock mechanics and rock engineering, 33:1, 31 (2000)

13. E, Komurlu, A. Kesimal, S. Demir , Geomech. Eng. 10:6, 775 (2016)

14. B. Liu, R.S. Yang D.M.I. Guo, Chinese journal of rock mechanics and engineering, 23:14, 2402 (2016)

15. C.Y. Liu , B.X. Huang, X.M. Chang, Journal of china university of mining and technology, 37:6, 734 (2008)

16. S.H. Liu, Z.H. Qin, J.F. Lou", Chinese journal of rock mechanics and engineering. 33:10, 2064 (2014)

17. Y.Y. Liu, S.R. Ye, P. Jiang, Z.H. Huang, H. Chen, Z.J. Du, Geomatics and information science of wuhan university, 39:10, 1135 (2014) 
18. Z.L. Mu, H. Wang, P. Peng, Z.J. Liu, X.C. Yang, Journal of mining \& safety engineering, 30:6, 841 (2013)

19. M Mohammadi, H. Tavakoli, Geomech. Eng., 9:1, 115 (2015)

20. Y. Ning, Journal of china coal society, 34:1, 50 (2009)

21. K, Panaghi, A. Golshani, T. Takemura, Geomech. Eng., 9:6, 793 (2015)

22. W.H. Pen, A.H. Lu, J Min safety eng, 25:2, 213 (2008)

23. M. Prudencio, M van SJ, International journal of rock mechanics and mining science, 44, 890 (2007)

24. Y.M. Song, Y. Zhang, H.L. Xu, X.F. Li, Y.F. Wang,Chinese journal of rock mechanics and engineering, 37:8, 1 (2018)

25. L. Selcuk, Geomech. Eng., 17, 597 (2019)

26. T. Tjongki, W.F. Kang, Chinese journal of rock mechanics and engineering, 10:4, 299 (1991)

27. T. Tjongkie, Chinese journal of rock mechanics and engineering, 1:1, 1 (1982)

28. P. Valkó, M. Economides, International journal of rock mechanics and mining sciences, 31:03, 221 (1994)

29. A. Vyazmensky, D. Elmo, Rock mechanics \&Rock engineering, 43:5, 533(2010)

30. T. Ge, D.R. Wang, Y.S. Lu, M.Y. Wang, O.H. Qian, “Chinese journal of rock mechanics and engineering, 26:5, 951 (2007)

31. J.C. Wang, Journal of china coal society, 32:8, 785(2007)

32. W. Changxiang, S. Baotang, C.Juntao, W. Tong, Z.Jiang, Y. Liu, Y. Li, Geomech Eng. 20:6, 485 (2020)

33. W. Brace, J. Byerlee, Science, 153:3739, 990 (1966)

34. C.D. Xie, X.L. Lei, X.P. Wu, H. Fu, Z.Y. Xiong, X.L. Hu, S. Li, Science china: earth sciences (2015)

35. H.L. Xing, A. Makinouchi, Computer methods in applied mechanics and engineering, 191, 3, 4193 (2002)

36. H.L. Xing, A. Makinouchi, P. Mora, Physics of the earth and planetary interiors, 163, 1, 106 (2007)

37. Q. Yao, H.L. Xing, X.W. Xu, W. Zhang, J. Liu, Seismology and geology, 40:1, 171 (2018)

38. H. Yossef, Journal of geotechnical and geoenvironmental engineering, 129:8, 697 (2003)

39. W.J. Yu, G.S. Wu, H. Liu, Journal of china coal society, 43:10, 2668 (2018)

40. Y .Yuan, S.H. Tu, X.T. Ma, Journal of mining \& safety engineering, 29:1, 21 (2012)

41. Z.Jinhai, Y.Liming, G.Weijia, Rock Mechanics and Rock Engineering, 51:8, 2355 (2018)

42. Z.Jinhai, J.Ning, Y. Liming, B. Li-yang, Bulletin of Engineering Geology and the Environment, 78:5, 3815 (2019)

43. Z. Jinhai, Z.Xinguo, J. Ning, Bulletin of Engineering Geology and the Environment (2020)

44. Z. Jinhai, L. Bo, C. Juntao, J. Ning, Arabian journal of geosciences (2020)

45. J.P. Zuo, Y. Chen, F. Cui, Journal of china university of mining and technology, 47:1, $81(2018)$ 